\title{
ON FUNDAMENTAL SEQUENCES OF TRANSLATES
}

\author{
R. A. ZALIK
}

ABSTRACT. We find Müntz-type theorems for sequences of the form $\left\{f\left(t+c_{n}\right)\right\}$ or $\left\{\exp \left(-c_{n} t\right) f(t)\right\}$ on $[0, \infty)$.

Let $R$ denote the set of real numbers, and $R^{+}$the set of nonnegative real numbers. Given a function $f(t)$, by $F(x)$ we shall denote its Fourier transform; thus if $f(t)$ is integrable on $R, F(x)=\int_{R} \exp (x t i) f(t) d t$.

A classical theorem of $\mathrm{N}$. Wiener affirms that if $f(t)$ is in $L_{2}(R)$, and $T(f)$ is the linear span of the set of functions of the form $f(t+c)$, with $c$ real, then $T(f)$ is dense in $L_{2}(R)$ if, and only if, $F(x) \neq 0$ a.e. in $R$ (cf. [1, p. 100, Theorem 12]). Motivated by Wiener's theorem, we found in [2] necessary and sufficient conditions for sequences of the form $\left\{f\left(t+c_{n}\right)\right\}$ or $\left\{\exp \left(c_{n} t i\right) f(t)\right\}$ to be fundamental in $L_{2}(R)$. The purpose of this paper is to consider the same problem on $R^{+}$.

Let $E$ denote one of the spaces $L_{p}\left(R^{+}\right)(p>1)$ or $C_{0}\left(R^{+}\right)$(the space of functions continuous on $R^{+}$that vanish at infinity, endowed with the uniform norm). Given a function $f(t)$, by $E(f)$ we shall denote the set of functions in $E$ that vanish wherever $f(t)$ vanishes. For a given sequence $\left\{c_{n}\right\}$, let $T(\varepsilon)$ denote the series $\Sigma_{c_{n} \neq 0}\left|c_{n}\right|^{-\varepsilon}$. With this notation we can state our first result.

THEOREM 1. Let $f(t)$ be a continuous function in $E$, not identically zero; let $\left\{c_{n}\right\}$ be a sequence of distinct complex numbers such that $\operatorname{Re}\left(c_{n}\right)>\delta\left|c_{n}\right|$, for some $\delta>0$. Let $f_{n}(t)=f(t) \exp \left(-c_{n} t\right)$. Then $\left\{f_{n}(t)\right\}$ is fundamental in $E(f)$ if, and only if, $T(1)$ is divergent.

Let $S$ represent one of the spaces $L_{p}[0,1](p>1)$, or $C[0,1]$ (the space of functions continuous on $[0,1]$ endowed with the uniform norm), and let $S(g)$ be the set of functions in $S$ that vanish wherever $g(x)$ vanishes. Making the change of variable $x=\exp (-t)$ we readily see that Theorem 1 is equivalent to

Theorem 2. Let $g(x)$ be a continuous function in $S$, not identically zero; let $\left\{c_{n}\right\}$ be a sequence of distinct complex numbers such that $c_{0}=0$, and $\operatorname{Re}\left(c_{n}\right)>\delta\left|c_{n}\right|$, for some $\delta>0$. Then $\left\{g(x) x^{c_{n}}\right\}$ is fundamental in $S(g)$ if, and only if, $T(1)$ diverges.

Remark. For $f(t)=1$, Theorem 1 reduces to a result of M. M. Crum [3]; it is clear, however, that Crum's theorem is equivalent to that of Müntz-Szász.

Received by the editors June 29, 1979.

1980 Mathematics Subject Classification. Primary 42A65; Secondary 41A30, 42A38.

Key words and phrases. Approximation by sequences of translates, entire functions of exponential type, holomorphic Fourier transform, Fourier-Stieltjes transform, inversion, Parseval's formula, PaleyWiener, Hahn-Banach, Riesz representation theorem. 
For sequences of translates we have the following:

Theorem 3. Let $\left\{c_{n}\right\}$ be a sequence of distinct complex numbers. Assume $f(z)$ is a not identically zero entire function of exponential type $a$, square integrable on $R$, and for some $\delta>0$ define $q(z)=\exp (-\delta z) f(z)$, and $q_{n}(t)=q\left(t+c_{n}\right)(t$ real $)$; then for $\left\{q_{n}(t)\right\}$ to be fundamental in $E$ it suffices that $T(\varepsilon)$ be divergent for some $\varepsilon>1$. If in addition $\operatorname{Re}\left(c_{n}\right)>0$ for all $n$, then the divergence of $T(1)$ is necessary for $\left\{q_{n}(t)\right\}$ to be fundamental in $E$.

The gap between the necessary and sufficient conditions in Theorem 3 can be eliminated by considering a slightly different function, and imposing restrictions on the sequence $\left\{c_{n}\right\}$; thus we have:

THEOREM 4. Let $\left\{c_{n}\right\}$ be a sequence of distinct complex numbers, bounded away from zero, with $\operatorname{Im}\left(c_{n}\right)<0, \operatorname{Re}\left(c_{n}\right) \geqslant 0$, and such that $\left\{\operatorname{Re}\left(c_{n}\right)\right\}$ is a bounded sequence. Let $f(z)$ and $q(z)$ be as in Theorem 3; let $h(z)=\exp ($ iaz $) q(z)$, and $h_{n}(t)=h\left(t+c_{n}\right)(t$ real $)$. Then for $\left\{h_{n}(t)\right\}$ to be fundamental in $E$, it is necessary and sufficient that $T(1)$ be divergent.

EXAmple. The function $f(z)=(\sin z) / z$ satisfies the hypotheses of Theorems 3 and 4.

Applying Theorem 4 with $a=\delta=1$ and $\operatorname{Re}\left(c_{n}\right)=1$, and eliminating redundant factors, we obtain the following:

COROLlary. Let $f(z) \neq 0$ be a square-integrable entire function of exponential type 1 , and let $\left\{\lambda_{n}\right\}$ be a strictly increasing sequence of strictly positive real numbers. Then the sequence $\left\{\exp (-t) f\left(t-i \lambda_{n}\right)\right\}$ is fundamental in $E$ if, and only if, $T(1)$ is divergent.

REMark. The set of functions $f(z)$ that satisfy the hypotheses of Theorems 3 and 4 is characterized by a well-known theorem of Paley and Wiener (cf. [4, p. 13, Theorem $X$ ] or Boas [5, p. 103, 6.8.1]).

Proof of Theorems 1 and 2. Sufficiency. We prove the sufficiency for Theorem 2. It suffices to assume that $S$ is the set $C_{0}[0,1]$, of continuous functions that vanish at 0 . Assume $T(1)$ is divergent and let $I$ be the set of points of $(0,1]$ at which $g(x)$ does not vanish. By the theorem of Hahn-Banach we know it suffices to show that any linear functional on $C_{0}[0,1]$ that annihilates the functions $g(x) x^{c_{n}}, n=$ $1,2,3, \ldots$, is identically zero. Applying the Riesz representation theorem we readily conclude that the assertion is equivalent to showing that if $\mu$ is a (finite) complex-valued measure with support in $I$ such that

$$
\int_{0}^{1} x^{c_{n}} g(x) d \mu(x)=0, \quad n=1,2, \ldots,
$$

then $\mu=0$. However ( 1 ) is equivalent to

$$
\int_{0}^{1} x^{c_{n}} d \nu(x)=0, \quad n=1,2, \ldots,
$$

where $d \nu(x)=g(x) d \mu(x)$, and the theorem of Müntz-Szász implies that $\nu=0$. 
Hence $\nu(A)=\int_{A} g(x) d \mu(x)=0$ for every measurable set $A$. Since $g(x) \neq 0$ on $I$, the conclusion follows.

Necessity. We prove the necessity for Theorem 1 . Let $p>1$ be given, and let $q$ be such that $p^{-1}+q^{-1}=1$. Since $f(t)$ is continuous and not identically zero, there is a closed interval $[\alpha, \beta], \alpha>0$, on which $f(t)$ does not vanish. Assume (1) is convergent; then, by a theorem of Luxemburg and Korevaar [6, p. 30, Theorem 5.2], there is a function $g(t)$ in $C^{\infty}(R)$ with supporting interval $[\alpha, \beta]$, such that the function $G(z)=\int_{\alpha}^{\beta} \exp (-i z t) g(t) d t$ vanishes at the points $-i c_{n}$, i.e.

$$
\int_{\alpha}^{\beta} \exp \left(-c_{n} t\right) g(t) d t=0, \quad n=0,1,2, \ldots
$$

Thus, if $g^{*}(t)=g(t) / f(t)$, it is clear that $g^{*}(t)$ is in $L_{q}(\alpha, \beta)$, is not equivalent to zero, and

$$
\int_{\alpha}^{\beta} f_{n}(t) g^{*}(t) d t=0, \quad n=0,1,2, \ldots
$$

Thus $\left\{f_{n}(t)\right\}$ cannot be fundamental in $L_{p}[\alpha, \beta]$, whence the conclusion readily follows, bearing in mind that $C[0,1]$ is dense in $L_{p}[0,1]$ for every $p>1$. Q.E.D.

Proof of Theorem 3. Sufficiency. It clearly suffices to assume that $E=C_{0}\left(R^{+}\right)$. Let $T(\varepsilon)$ be divergent for some $\varepsilon>1$, and assume $\mu$ is a (finite) complex-valued measure with support in $R^{+}$, such that

$$
\int_{R} q\left(t+c_{n}\right) d \mu(t)=0, \quad n=0,1,2, \ldots
$$

Setting $d \nu(t)=\exp (-\delta t) d \mu(t)$, we readily conclude that

$$
\int_{R} f\left(t+c_{n}\right) d v(t)=0, \quad n=0,1,2, \ldots
$$

Let $F$ and $G$ be the Fourier transforms of $f$ and $\nu$ respectively, and let

$$
r(z)=(2 \pi) \int_{R} f(t+z) d \nu(t) .
$$

We know from $[5$, p. 103, (6.8.2)] that, for real $t,|f(t+z)|<K \exp (a|y|)$, where $y$ is the imaginary part of $z$, and $K$ is a constant independent of $t$. Since $\nu$ is a finite measure, we conclude that (4) is defined for all complex $z$; thus, applying the theorems of Morera and Fubini, we readily see that (4) defines an entire function of exponential type. Since (from (3)), $r(z)$ vanishes at the points $c_{n}$, we conclude from [5, p. 17, 2.5.18] that $r(z)$ vanishes identically. Since (by Parseval's formula), $r(z)$ is the Fourier transform of $F(-t) G(t)$ (cf. Katznelson [7, p. 132], bearing in mind that we define Fourier transforms in terms of $\exp (z t i)$, and not in terms of $\exp (-z t i)$, and that the Fourier transform of $f(t+z)$ is $F(\xi) \exp (-\xi z i))$, we conclude that this product is equivalent to zero. Since $F(t)$ cannot be equivalent to zero (for this would imply that $f(z)$ vanishes identically), we see that $G(t)$ vanishes on a nondenumerable subset of the real line. However, since $d \nu(t)=\exp (-\delta t) d \mu(t)$, with $\mu$ bounded and with support in $R^{+}$, we readily see that $G$ is holomorphic in $\operatorname{Im}(z)<\delta$. Since the real line is interior to this domain, we conclude that $G$ vanishes identically thereon. Since the real and imaginary parts of $\nu$ are bounded, 
they can be represented as the difference of two finite and positive measures. Applying now Bochner's theorem on the uniqueness of the Fourier-Stieltjes transform (cf. Cotlar [8, p. 523, Theorem 3.1.9(c)]), we readily see that $\nu=0$; hence (as in the proof of Theorems 1 and 2) also $\mu=0$, and the conclusion follows.

Necessity. Let $f^{*}(z)=f(z)$ if $\operatorname{Re}(z) \geqslant 0$, and $f^{*}(z)=0$ elsewhere, and let $q^{*}(z)=$ $\exp (-\delta z) f^{*}(z)$. Since $f$ is in $L_{2}(R)$, it is clear that also $f^{*}$ is in $L_{2}(R)$, and we readily see that $Q^{*}$ (the Fourier transform of $q^{*}$ ) is holomorphic in $\operatorname{Im}(z)<\delta$. Since $Q^{*}(t)$ is clearly not the zero function, there is an interval $[\alpha, \beta]$ on which $Q^{*}(-t)$ does not vanish. Assume that (1) converges. Then applying [6, p. 30, Theorem 5.2], we see there is a function $g(t)$ in $C^{\infty}(R)$, with supporting interval $[\alpha, \beta]$, such that the function $G(z)=\int_{\alpha}^{\beta} \exp (z t i) g(t) d t$ vanishes at the points $c_{n}$. Clearly $G(z)$ is an entire function; moreover, since $Q^{*}(-t)$ does not vanish on $[\alpha, \beta]$, setting $g_{1}(t)=$ $g(t) / Q^{*}(-t)$, on $[\alpha, \beta]$, and $g_{1}(t)=0$ elsewhere, we have:

$$
G(z)=\int_{R} \exp (z t i) Q^{*}(-t) g_{1}(t) d t .
$$

It is clear that $g_{1}(t)$ is in $C^{\infty}(R)$ and has bounded support; thus $g_{1}^{\prime}(t)$ is bounded, and we infer that $g_{1}(t)$ satisfies a Lipschitz condition of order 1. Applying now a theorem of Bernstein (cf. [7, p. 32]) we conclude that $g_{1}(t)$ has an absolutely convergent Fourier series on any interval of the form $[\alpha-\eta, \beta+\eta], \eta>0$.

Making an obvious change of variable, and applying [5, p. 106, 6.8.11], it is easy to see that $G_{1}$ (the Fourier transform of $g_{1}$ ) is in $L_{1}(R)$. Since $g_{1}$ is in $L_{1}(R)$ we also know that $G_{1}$ is in $C_{0}(R)$ and is therefore bounded, and we readily infer that $G_{1}$ is in $L_{q}(R)$ for every $q \geqslant 1$. Applying Parseval's formula [4, p. 2, (1.09)] and noting that $2 \pi g_{1}(t)$ is the Fourier transform of $G_{1}(-x)$, we see that for $z$ real and positive,

$$
2 \pi G(z)=\int_{R^{+}} q^{*}(x+z) G_{1}(x) d x
$$

Since it is readily seen that the right-hand member of (5) is holomorphic in $\operatorname{Re}(z)>0$ and continuous in $\operatorname{Re}(z) \geqslant 0$, (5) holds identically in the latter region.

Since $G(z)$ vanishes at the points $c_{n}$, we see from (5) that

$$
\int_{R^{+}} q^{*}\left(x+c_{n}\right) G_{1}(x) d x=0, \quad n=0,1,2, \ldots
$$

thus the sequence $\left\{q^{*}\left(x+c_{n}\right)\right\}$ cannot be fundamental in any $L_{p}\left(R^{+}\right)$. This implies that also $\left\{q_{n}\right\}$ cannot be fundamental thereon, whence the conclusion follows. Q.E.D.

Proof of Theorem 4. Sufficiency. Assume there is a (finite) complex-valued measure $\mu$, with support in $R^{+}$, such that

$$
\int_{R} h\left(t+c_{n}\right) d \mu(t)=0, \quad n=0,1,2, \ldots
$$

Setting $d \nu(t)=\exp (-\delta t) d \mu(t)$, we readily see that (6) is equivalent to

$$
\int_{R} \exp \left[a\left(t+c_{n}\right) i\right] f\left(t+c_{n}\right) d \nu(t)=0, \quad n=0,1,2, \ldots
$$


Let $F$ and $G$ denote the Fourier transforms of $f$ and $\nu$ respectively, and let

$$
r(z)=\int_{R} \exp [a(t+z) i] f(t+z) d \nu(t) .
$$

Proceeding as in the proof of Theorem 3, we readily see that $r(z)$ is holomorphic and bounded in the region $\operatorname{Im}(z)<0$. We also know from (7) that $r(z)$ vanishes at the points $c_{n}$. Since the function $v(z)=-i(1+z) /(1-z)$ transforms the interior of the unit circle conformally onto the region $\operatorname{Im}(z)<0$, we see that the function $r[v(z)]$ is in the Hardy space $H^{\infty}$, and vanishes at the points $\lambda_{n}=\left(c_{n}+i\right) /\left(c_{n}-i\right)$. Proceeding as in [9, p. 337], we conclude that $r(z)$ vanishes identically on $\operatorname{Im}(z)<$ 0 , and $r(z)$ being an entire function, this implies that it vanishes everywhere on the complex plane. Since (by Parseval's theorem) $2 \pi r(z)$ is the Fourier transform of $F(a-t) G(t)$, we conclude that this product is equivalent to zero. Employing the same argument that was applied in the proof of Theorem 3, it is now easy to see that $\mu=0$, whence the conclusion follows.

The proof of the necessity follows by applying the necessary part of Theorem 3 to $\exp (i a z) f(z)$ instead of to $f(z)$. Q.E.D.

\section{REFERENCES}

1. N. Wiener, The Fourier integral and certain of its applications, Cambridge Univ. Press, Cambridge, 1933; reprint, Dover, New York, 1958.

2. R. A. Zalik, On approximation by shifts and a theorem of Wiener, Trans. Amer. Math. Soc. 243 (1978), 299-308.

3. M. M. Crum, On the theorems of Müntz and Szász, J. London Math. Soc. 31 (1956), 433-437; Corrigendum and Addendum, ibid. 32 (1957), 512.

4. R. E. A. C. Paley and N. Wiener, Fourier transforms in the complex domain, Amer. Math. Soc. Colloq. Publ., vol. 19, Amer. Math. Soc., Providence, R. I., 1934; 3rd printing, 1978.

5. R. P. Boas, Jr., Entire functions, Academic Press, New York, 1954.

6. W. A. J. Luxemburg and J. Korevaar, Entire functions and Müntz-Szász type approximation, Trans. Amer. Math. Soc. 157 (1971), 23-37.

7. Y. Katznelson, An introduction to harmonic analysis, 2nd ed., Dover, New York, 1976.

8. M. Cotlar and R. Cignoli, An introduction to functional analysis, American Elsevier, New York, 1974.

9. W. Rudin, Real and complex analysis, 2nd ed., McGraw-Hill, New York, 1974.

Department of Mathematics, Auburn University, Auburn, Alabama 36830 